\title{
IMPACTS OF POWER CONTROL ON OUTAGE PROBABILITY IN CDMA WIRELESS SYSTEMS
}

\author{
Kenji Leibnitz \\ University of Würzburg, Dept. of Computer Science, Am Hubland, 97074 Würzburg, Germany \\ leibnitz@informatik.uni-wuerzburg.de
}

\begin{abstract}
In this paper we analyze the power control loops on the reverse link of a CDMA system. We obtain an expression for the transmission power of a mobile station and its signal-to-interference ratio (SIR) received at the base station. This allows the derivation of outage probability, which is the probability for not fulfilling the SIR requirements. We will show that power control in the North-American IS-95 system is very robust and that the number of users in the cell does not have much influence on outage. Furthermore, we compare outage which is an event at the receiver with the mobile station exceeding its maximum transmission power.
\end{abstract}

Keywords: CDMA, power control, signal-to-interference ratio, outage probability.

\section{INTRODUCTION}

Wideband Code Division Multiple Access (W-CDMA) is the upcoming RF technology for future mobile communication systems, like Universal Mobile Telecommunications System (UMTS) or IMT-2000. This is mainly due to its superior capacity compared to second generation systems employing F/TDMA. In CDMA the transmitted signal is spread over the frequency bandwidth by modulating each user's data signal with a pseudo-noise carrier of much higher frequency. Since the signals appear like noise over the channel, all other users in the cell constitute to a certain level of interference.

This limitation leads to the necessity of controlling the interference induced by other users to a minimum. Mobile stations (MS) will be located in the cell at varying distances from the base transceiver station (BTS). It has to be avoided that a mobile near the BTS transmits at a too high level and causes too much interference for other MS farther away ("near-far" problem). Additionally, due to shadowing and multi-path fading as well as fluctuations in user traffic there will be variations in the received signal strength. This is overcome by a tight power control performed on the reverse link (mobile-to-base station path). 
With this power control algorithm the BTS tries to perform a balancing of the received signal-to-interference ratio (SIR) of all users in the cell.

The performance of closed loop power control based on local SIR estimates has been studied by several authors. In [14] an analytical model was constructed containing an inner loop based on SIR processing and an outer loop based on frame error performance. Simulation studies of single and multi-cell systems were conducted in [1] and the dependence of signal and interference statistics on step size and processing delays was examined. A similar approach was taken in [2], where the impact of update rate, loop delay and vehicle speed on the bit error performance was examined in simulations. The effects of power control non-idealities on performance were investigated in [3] and [8].

The model proposed here is based on the one presented in [4] and is a Markov state space representation of the closed loop power control which allows direct computation of the statistics of the MS transmit power. We will investigate how the power control loops affect the SIR and derive an expression for outage probability. Furthermore, we will show the relationship between outage, which is an event occurring at the BTS and the event of the mobile station exceeding its maximum transmission power.

This paper is organized as follows. Section 2 gives an overview of the closed loop power control which is implemented in the IS-95 system [11]. In Section 3 we present the system parameters and Gaussian channel and derive our analytical Markov chain model. Based on this model, we will discuss the impacts of power control on outage probability in Section 4. This paper is concluded in Section 5 by giving an outlook on future work.

\section{MODEL OF THE IS-95 CDMA SYSTEM}

In CDMA systems it is essential that all users are received with nearly equal strength at the BTS in order to be demodulated and decoded correctly. To overcome the near-far problem, several mechanisms to control the MS transmit power take place in the IS-95 standard at the base and mobile stations.

In open loop power control the MS uses the received signal strength on the forward link as estimation for the path loss and sets its transmit power accordingly. Contrary to that, closed loop power control works in a tight cooperation between mobile and base station to overcome fluctuations on the traffic channel. The closed loop itself consists of an inner loop and outer loop. Within the inner loop, the BTS continually monitors the link quality of the reverse link in terms of received SIR and compares it with a certain threshold. If the received value is too high, then the MS is told to decrease its power. On the other hand, if it is too low, the link quality is not good enough and a "power-up" command is sent. This power update is performed every 1.25 $m s$. The power control command itself consists of one bit of information that 
is multiplexed on the traffic channel after the convolutional encoding and is therefore not error protected. The transmission of a single bit also results in the fact that there are only commands for increasing or decreasing, but none for maintaining a certain power level.

After every 16 such inner loop cycles, one frame has been transmitted and the power control algorithm enters the outer loop. Its main goal is to maintain an acceptable frame error rate (FER) by readjusting the SIR threshold of the inner loop after every frame. The interaction between inner and outer loop is illustrated in Fig. 1.

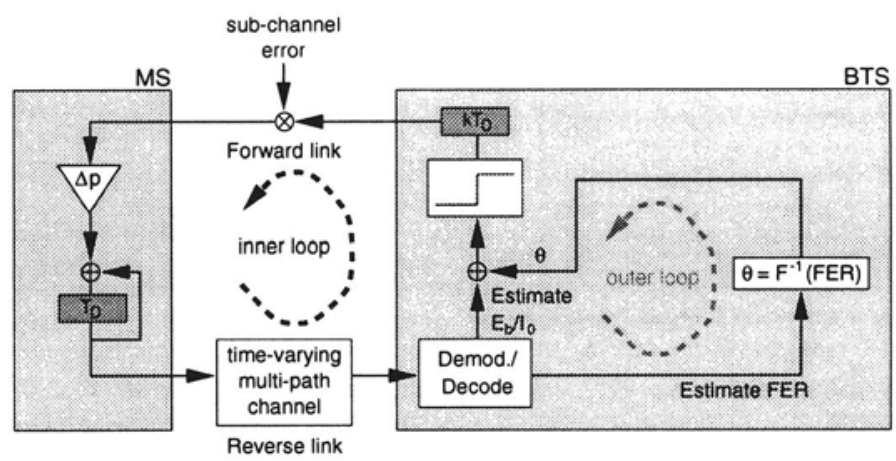

Figure 1 CDMA reverse link closed loop power control

\section{ANALYSIS OF CDMA POWER CONTROL}

In this section we derive an analytical model of the reverse link power control loops which will permit the computation of the transmit power of an arbitrary MS. Our model is based on a Markov chain which is discrete in time $(1.25 \mathrm{~ms}$ steps) and discrete in state space. In the IS-95 system, the MS transmit power is limited to the range between -50 and $23 \mathrm{dBm}$ and the update step size is $1 d B$. In the following, we will denote the mobile's power with the variable $j=0, \ldots, J$, where $J$ is the total number of possible steps within the range given above.

\subsection{System Parameters and Gaussian Channel}

Let an MS be located at a distance $x$ from the BTS and $\hat{S}$ be its transmission power at an observed time instant. On the path to the base station, the signal power is being attenuated by propagation loss $L(x)$ [9], shadow fading, and multi-path fading and is received with power $T$ at the BTS. The received SIR is given by the bit-energy-to-noise ratio $\left(E_{b} / N_{0}\right)$ denoted by the variable $\epsilon$. Note that variables in linear space will be given as $\hat{\chi}$ which is $\chi=10 \log \hat{\chi}$ in $\mathrm{dB}$. 


$$
\hat{\epsilon}=\frac{\hat{G}_{s} \hat{T}}{\sum_{i=1}^{k-1} \hat{T}_{i} \nu_{i}+N_{0} W}
$$

The spreading gain of the system is $\hat{G}_{s}=W / R$ with a data bitrate of $R=9.6$ kbps and frequency spectrum of $W=1.25 \mathrm{Mhz}$. The term $N_{0}$ is the thermal noise power density. By introducing $k_{\text {pole }}=W /\left(R m_{\hat{\epsilon}} \rho\right)+1$ as the pole capacity [12] of the system for a mean SIR $m_{\hat{\epsilon}}$ we can eliminate the dependency on the received power levels $\hat{T}$ and can rewrite Eqn. (1) as

$$
\hat{\epsilon}=\hat{G}_{s} \frac{\hat{S} \hat{L}(x)}{\hat{N}_{0}} \hat{\varphi}(k) \quad \Rightarrow \quad \epsilon=G_{s}+S+L(x)+\varphi(k)-N_{0} .
$$

The multi-access interference (MAI) induced by the other users in the same cell is represented by $\hat{\varphi}(k)=\left(k_{\text {pole }}-k\right) /\left(k_{\text {pole }}-1\right)$ and is a function of the number of users $k$. If the system supports only a single user, the term $\hat{\varphi}$ will be one and there will be no interference from other users. However, if $k$ is near $k_{\text {pole }}$ then the MAI causes that the SIR approaches zero. Note that at this point we don't know the exact value of $m_{\hat{\epsilon}}$. However, for our purposes it is sufficient to assume a fixed $k_{\text {pole }}$ and observe the loading percentage of the cell.

We will use an Additive White Gaussian Noise (AWGN) channel model where the total attenuation caused by fading is considered to be an i.i.d. Gaussian random variable $C$ with mean $\mu_{C}$ and standard deviation $\sigma_{C}$. We can write $\mu_{C}=G_{s}+L(x)+\varphi(k)-N_{0}$ in our case as the sum of all non-random components of Eqn. (2). We can now express $\epsilon$ as the sum of the random variables $S$ and $C$. In the following we will derive the distribution of $S$.

\subsection{Closed Loop Power Control}

As mentioned in Section 2, the closed loop power control in IS-95 consists of the interworking of inner and outer loop. The following section will describe how both loops are taken into account in our model.

\subsubsection{Inner Loop}

In the inner loop the received SIR level $\epsilon$ is compared with the target threshold $\theta$ of the outer loop whether to increase or decrease the signal strength. Thus, the probability for a power-up command is $p_{u}=\operatorname{Pr}(\epsilon<\theta)$.

Using the properties of the Gaussian distribution, the probability for a power up command $p_{u}$ under the condition that the MS is transmitting at power level $j$ and the outer loop threshold is $i$ can be given as

$$
p_{u}(i, j)=\operatorname{Pr}(C \leq i-j \mid S=j, \theta=i)=\frac{1}{2}+\frac{1}{2} \operatorname{erf}\left(\frac{i-j-\mu_{C}}{\sqrt{2} \sigma_{C}}\right) .
$$


Since it is only possible to increase or decrease the power, the probability for a power-down command is $p_{d}(i, j)=1-p_{u}(i, j)$.

As previously mentioned, the power control command is transmitted unprotected. We will therefore model the forward link channel as a binary symmetrical channel with a bit error probability $p_{b}$. It is well known [15] that the probability of bit error in a QPSK modulated channel can be approximated by $p_{b}=\frac{1}{2} Q\left(\sqrt{E_{b} / N_{0}}\right)$.

\subsubsection{Outer Loop}

The outer loop is performed after every frame, i.e., 16 inner loop cycles. Its purpose is to update the SIR threshold to achieve an acceptable link quality in terms of frame error rate. Whereas the inner loop is specified in IS-95, the algorithm for the outer loop is up to the manufacturer. In the following we will use a simple algorithm similar to the one presented in [10].

Let $\theta$ be the threshold level at a certain frame. If the frame is in error, the threshold is increased by $K d B$, e.g. $K=5$, otherwise it is decreased by $1 d B$. The frame is considered to be in error if at least one bit in the frame is in error, which can occur independently. Therefore, for $N=192$ bits in a frame and the bit error probability $p_{b}$ given above, the probability for increasing the SIR threshold is $q_{u}(i)=1-\left(1-p_{b}(i)\right)^{N}$ and for decreasing is $q_{d}(i)=1-q_{u}(i)$. It is assumed that the threshold will be limited by a maximum value $M$.

\subsubsection{Markov Chain Model}

To model the power control loops we will use a Markov chain with twodimensional states $s(i, j), i=1, \ldots, M, j=1, \ldots, J$. The first index $i$ describes the SIR threshold value and $j$ is the power level at which the MS is transmitting. The state transitions to and from a state $s(i, j)$ of this Markov Chain are illustrated in Fig. 2.

The power level will be increased and decreased with $p_{u}(i, j)$ and $p_{d}(i, j)$ in Eqn. (3), respectively. Since the inner loop is performed more frequently than the outer loop, where a threshold update is done every 16 th cycle, the transitions with the same SIR threshold are weighted with $\frac{15}{16}$. The transitions from $s(i, j)$ to states with other thresholds must consider the probability for threshold updates $q_{u}(i)$ and $q_{d}(i)$. Therefore, the following transition probabilities are used.

$$
\begin{array}{lll}
\alpha(i, j)=\frac{15 p_{u}(i, j)}{16} & \beta(i, j)=\frac{15 p_{d}(i, j)}{16} & \gamma(i, j)=\frac{p_{d}(i, j) q_{d}(i)}{16} \\
\delta(i, j)=\frac{p_{u}(i, j) q_{d}(i)}{16} & \xi(i, j)=\frac{p_{d}(i, j) q_{u}(i)}{16} & \zeta(i, j)=\frac{p_{u}(i, j) q_{u}(i)}{16}
\end{array}
$$

Note that special care has to be taken at the range boundaries for $i \in\{0, M\}$ and $j \in\{0, J\}$. We can now order all probabilities in a transition probability 
matrix $\mathcal{P}$, see Eqn. (4).

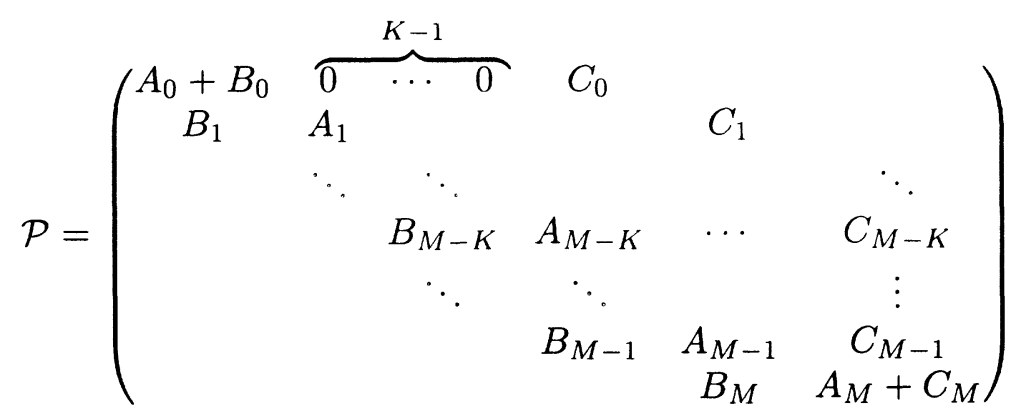

The matrices $A_{i}, B_{i}$, and $C_{i}$ can be given in the following way. If we define a matrix $P_{i}, i=1, \ldots, M$, by

$$
P_{i}=\left(\begin{array}{ccccc}
p_{d}(i, 0) & p_{u}(i, 0) & & & \\
p_{d}(i, 1) & & p_{u}(i, 1) & & \\
& \ddots & & \ddots & \\
& & p_{d}(i, J-1) & & p_{u}(i, J-1) \\
& & & p_{d}(i, J) & p_{u}(i, J)
\end{array}\right)
$$

then $A_{i}=\frac{15}{16} P_{i}, B_{i}=\frac{q_{d}(i)}{16} P_{i}$, and $C_{i}=\frac{q_{u}(i)}{16} P_{i}$. With Eqn. (4) it is now possible to compute the equilibrium state distribution $s(i, j)$. The stationary MS power distribution can be derived by the sum of all states with common second index and the distribution of the outer loop threshold by summation of the states with common first index, see Eqn. (5).

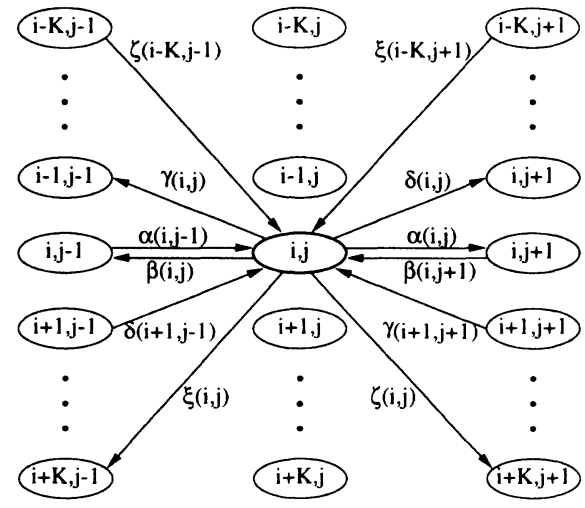

Figure 2 Markov Chain state transitions

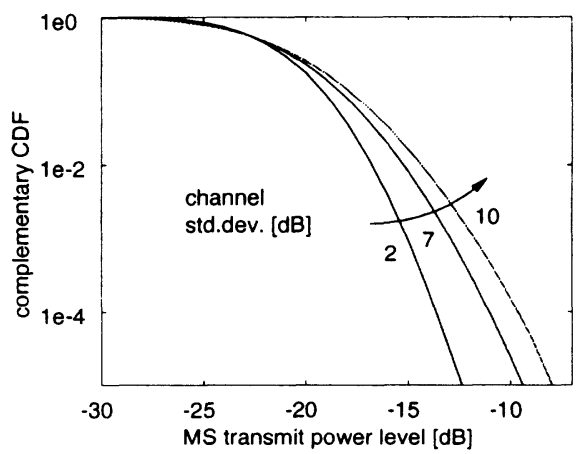

Figure 3 MS transmit power distribution 


$$
\operatorname{Pr}(S=j)=s(j)=\sum_{i=0}^{M} s(i, j) \quad \text { and } \quad \operatorname{Pr}(\theta=i)=\sum_{j=0}^{J} s(i, j)
$$

Figure 3 depicts the complementary cumulative distribution function (CDF) of the MS transmit power with $k=15$ users in the cell and the observed user at a distance of $x=2000 \mathrm{~m}$ from the BTS. It can be seen that when the channel gets worse, a higher transmit power is required.

We have performed computations of the mean and standard deviation of the random variable $S$ for varying parameters $k$ and $x$. It could be seen that variations of the traffic load had almost no impact on the statistics of $S$. However, the distance plays an important role on the transmit power. Fig. 4 shows that there is a logarithmic relationship between the mean transmit power $E[S]$ and the distance due to path loss. The standard deviation decreases with growing distance, since the MS will be transmitting almost deterministically with maximum power at the cell boundaries.

\section{IMPACTS OF POWER CONTROL ON OUTAGE}

So far we have derived an expression for the transmit power of the MS at a distance $x$ from the base station and $k$ other users in the cell. We will now use this description to analyze the performance of the system by investigating the effects of these two parameters on the probability of outage.
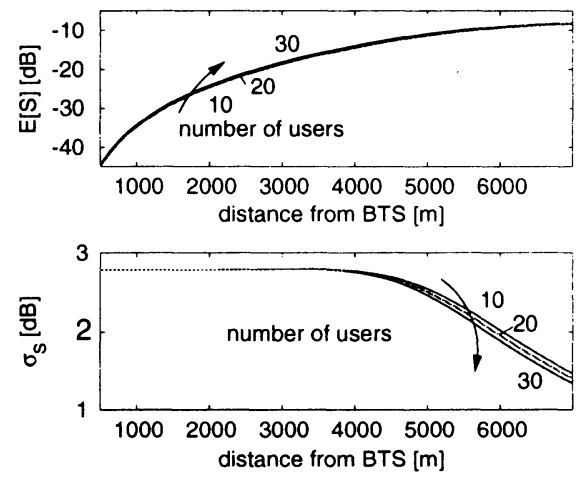

Figure 4 Mean and standard deviation of S

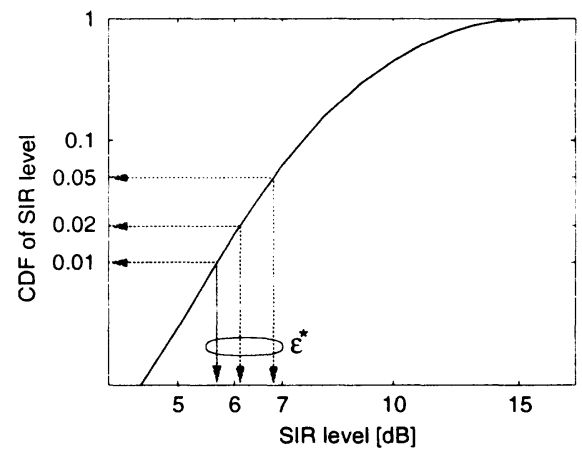

Figure 5 Cumulative SIR distribution

\subsection{Outage Condition}

The probability of outage is an important performance measure in a CDMA system $[12,13]$. It is defined as the probability that the SIR of a user lies below a minimum requirement threshold $\epsilon^{\star}$ for a certain amount of time, usually a 
few frames. Since the time scale is such small (about $100 \mathrm{~ms}$ ), it is assumed that the dynamics of the spatial user distribution can be neglected. The SIR is measured at the BTS, making outage an event occurring at the receiver, i.e., $P_{\text {out }}=\operatorname{Pr}\left(\epsilon<\epsilon^{\star}\right)$.

In Section 3.2 we described the variable $\epsilon$ as the sum of $S$ and $C$. With the MS transmit power in Eqn. (5) and $C$ being Gaussian, both distributions are now known. The distribution of $\epsilon$ can be computed in this case by using the total probability law and conditioning the transmit power for a fixed channel gain.

The resulting SIR distribution has the shape of a Gaussian distribution, a fact which has also been confirmed by simulation and empirical results [14]. For our purpose of determining the outage probability, the CDF of the SIR is more useful. For a certain minimum required link quality given by $\epsilon^{\star}$, we can find the corresponding probabilities to be below this level. In the same way, we can give the minimal SIR requirement for a maximum allowable outage probability (Fig. 5).

Since the distribution of $\epsilon$ still has the two parameters $x$ and $k$, we have investigated the dependence on these two values. Figure 6 shows the variations of the mean and standard deviation of $\epsilon$ as function of the distance. It can be seen that for distances less than $4 \mathrm{~km}$ the power control can keep the mean and standard deviation at nearly equal levels. With greater distance, however, the mean decreases due to path loss. The inability of the power control loops at this distance causes also that the standard deviation of the SIR increases at greater distances. Users at the cell boundaries will therefore experience a higher outage probability than users in the cell. Note also that up to about $4 \mathrm{~km}$ the load of the cell does not influence the distribution of SIR. Therefore, for an examination of outage probability the distribution of the users is only of minor importance.
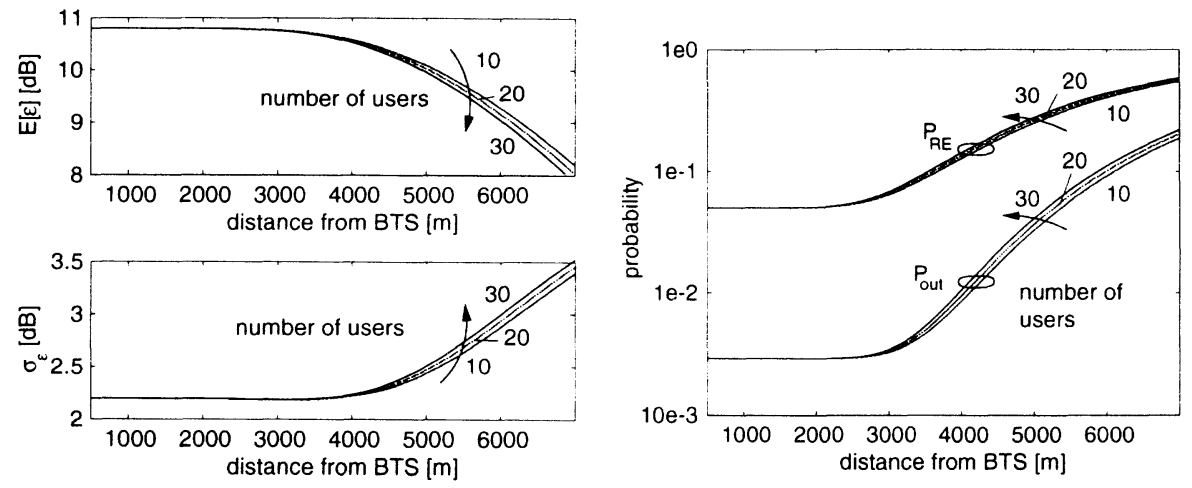

Figure 6 Mean and standard deviation of SIR

Figure $7 \quad P_{\text {out }}$ versus $P_{\mathrm{RE}}$ 


\subsection{Probability of Exceeding the Transmit Power Range}

Another term often used to describe outage probability is the probability of the MS exceeding its dynamic range since this will be the obvious cause for not being able to fulfill the SIR requirements $[7,12]$.

We obtain $P_{\mathrm{RE}}$ from our Markov chain model from the probability of powerup under the condition of transmitting at maximum power level $J$, i.e.,

$$
P_{\mathrm{RE}}=\operatorname{Pr}\left(S>S_{\max }\right)=\sum_{i=0}^{M}(\alpha(i, J)+\delta(i, J)+\zeta(i, J)) \operatorname{Pr}(\theta=i)
$$

Figure 7 shows both interpretations of outage probability. The curve computed for $P_{\text {out }}$ is as expected almost independent of the distance $x$ up to about $4 \mathrm{~km}$. Then it increases due to the higher propagation loss. The threshold value in this case was selected as $\epsilon^{\star}=6 d B$ which corresponds to a maximum tolerable FER. Furthermore, we have also plotted the probabilities for exceeding the dynamic range of the mobile transmitter. It can be seen that for increasing cell load both curves have a similar shape. However, the range exceeding probability $P_{\mathrm{RE}}$ always overestimates $P_{\text {out }}$ by a magnitude.

\section{CONCLUSION AND OUTLOOK}

In this paper we have presented a simple analytical model for the closed loop power control in a CDMA system. With our model, we obtain the distribution of the transmit power of the MS, as well as the SIR received at the base station from this mobile. We have shown that the power control algorithm currently implemented in the IS-95 system is robust enough to overcome the near-far problem and fluctuations in cell traffic. Furthermore, the often used relationship between the probability of exceeding the MS transmit power and outage prebability cannot be confirmed. However, both probabilities are functions of the transmit power, which is dependent on the distance of the MS to the base station and only to some extent by the number of users in the cell.

We have also seen that the examination of outage is quite straightforward in the stationary case. However, when observing the temporal fluctuations of the channel by considering a correlated fading channel, the computation of the power control loops will become a challenging task. Some research has already been done on the outage characterization over time $[5,6]$. In future work we will extend our model to include the temporal behavior of the channel, as well as examine power control in a multi-cell environment with soft-handoff. The performance analysis of CDMA systems becomes especially important, as future generation W-CDMA systems like UMTS will have a power control mechanism very similar to the one described in this paper. 


\section{Acknowledgments}

The author would like to thank Prof. P. Tran-Gia and N. Gerlich (University of Würzburg) and J.E. Miller (NORTEL Wireless Networks, Richardson) for the valuable discussions during the course of this work. Part of this work has been supported by NORTEL External Research.

\section{References}

[1] Ariyavisitakul, S. and Chang, L.F. (1993) Signal and interference statistics of a CDMA System with feedback power control. IEEE Trans. on Comm., vol. $41 \mathrm{nr} .11$.

[2] Chockalingam, A., Dietrich, P., Milstein, L.B., and Rao, R.R. (1998) Performance of Closed-Loop Power Control in DS-CDMA Cellular Systems. IEEE Trans. on Veh. Tech., vol. 47 nr. 3.

[3] D'Avella, R., Marizza, D., and Moreno, L. (1994) Power Control in CDMA Systems: Performance Evaluation and System Design Implications. Proc. of IEEE ICUPC, San Diego, CA.

[4] Leibnitz, K., Tran-Gia, P., and Miller, J.E. (1998) Analysis of the Dynamics of CDMA Reverse Link Power Control. Proc. of IEEE GLOBECOM, Sydney, Australia.

[5] Mandayam, N.B., Chen, P.-C., and Holtzman, J.M. (1998) Minimum Duration Outage for CDMA Cellular Systems: A Level Crossing Analysis. Journal of Wireless Pers. Comm.

[6] Mukherjee, S. and Viswanathan, H. (1998) Minimum duration outages for diversity systems. Proc. of IEEE GLOBECOM, Sydney, Australia.

[7] Patel, P.R., Goni, U.S., Miller, E., and Carter, P.P.S. (1996) A Simple Analysis of CDMA Soft Handoff Gain and its Effect on the Cell's Coverage Area. In Wireless Information Networks, Kluwer Academic, Boston.

[8] Pichna, R., Wang, Q., and Bhargava, V.K. (1993) Non-Ideal Power Control in DS-CDMA Cellular. Proc. of IEEE Pacific Rim Conf. on Comm., Computers and Signal Processing.

[9] Rappaport, T.S. (1996) Wireless Communications - Principles \& Practice. Prentice Hall, Upper Saddle River, NJ.

[10] Sampath, A., Kumar, P.S., and Holtzman, J.M. (1997) On setting reverse link target SIR in a CDMA system. Proc. of IEEE Veh. Tech. Conf., Phoenix, AZ.

[11] TIA/EIA/IS-95 (1995) Mobile station - Base station compatability standard for dualmode wideband spread spectrum cellular systems.

[12] Veeravalli, V.V., Sendonaris, A., and Jain, N. (1997) CDMA Coverage, Capacity and Pole Capacity. Proc. of IEEE Veh. Tech. Conf., Phoenix, AZ.

[13] Viterbi, A.M. and Viterbi, A.J. (1993) Erlang capacity of a power controlled CDMA system. IEEE Journal on Sel. Areas in Comm., vol. 11 nr. 6.

[14] Viterbi, A.J., Viterbi, A.M., and Zehavi, E. (1993) Performance of power-controlled wideband terrestrial digital communication. IEEE Trans. on Comm., vol. $41 \mathrm{nr} .4$.

[15] Yang, S.C. (1998) CDMA RF System Engineering. Artech House. 